\title{
Astérisque
}

\section{MEINOLF GECK}

\section{Representations of Hecke algebras at roots of unity}

Astérisque, tome 252 (1998), Séminaire Bourbaki, exp. no 836, p. 33-55

<http://www.numdam.org/item?id=SB_1997-1998_40_33_0>

(C) Société mathématique de France, 1998, tous droits réservés.

L'accès aux archives de la collection « Astérisque » (http://smf4.emath.fr/ Publications/Asterisque/) implique l'accord avec les conditions générales d'utilisation (http://www.numdam.org/conditions). Toute utilisation commerciale ou impression systématique est constitutive d'une infraction pénale. Toute copie ou impression de ce fichier doit contenir la présente mention de copyright.

\section{NumDam}

Article numérisé dans le cadre du programme Numérisation de documents anciens mathématiques http://www.numdam.org/ 


\title{
REPRESENTATIONS OF HECKE ALGEBRAS AT ROOTS OF UNITY
}

\author{
by Meinolf GECK
}

Hecke algebras arise naturally in the representation theory of finite or $p$-adic Chevalley groups, as endomorphism algebras of certain induced representations (see Carter [9], Lusztig [44], and the references there). They may also be viewed as quotients of group algebras of Artin-Tits braid groups, and then they can be used to construct invariants of knots and links (Jones [36]).

Another point of view - which we take in this talk - is to regard them abstractly as deformations of group algebras of finite Coxeter groups, depending on a parameter $u$. If we specialize this parameter to a root of unity, we obtain in general a non-semisimple specialized algebra. The degree of non-semisimplicity is measured in terms of a corresponding decomposition matrix, which records in which way the irreducible representations of the generic algebra split up under specialization.

The aim of this talk is to explain some of the main open problems about decomposition matrices of Hecke algebras, and to report on some significant recent advances in this area.

For applications to the representation theory of a finite Chevalley group $G$ over a field of characteristic $p$, the most interesting specializations are those where $u$ is mapped to a non-zero element in a field of positive characteristic $\ell \neq p$. By Dipper's theory of Hom functors [14], a special case of which we describe in Section 1, the corresponding decomposition matrix is a submatrix of the usual $\ell$-modular decomposition matrix of $G$.

A general factorization result, which we explain in Section 2, shows that our decomposition matrices can be obtained in two steps: one step from $u$ to a root of unity over $\mathbb{Q}$, and another step from characteristic 0 to characteristic $\ell$. A conjecture which was first formulated by James [32] for Hecke algebras associated to the symmetric group $\mathfrak{S}_{n}$ predicts that "nothing happens" in the second step, as long as $\ell$ is not too small. (James actually considers an enlargement of that algebra, namely the $q$-Schur algebra introduced by Dipper and himself in [17]; he also gives a precise bound for $\ell$.) A rigorous formulation of this conjecture, for finite Coxeter groups of any type, will be given in Section 3.

For Hecke algebras associated with $\mathfrak{S}_{n}$, Lascoux, Leclerc and Thibon [39] have conjectured a solution of the first step, by translating the original problem to that of computing 
the canonical basis (in the sense of Kashiwara and Lusztig; see [46]) of a certain highest weight module for an affine Kac-Moody algebra; see Theorem 4.3. In Sections 4 and 5 we explain the main ideas of Ariki's proof [2] of this conjecture, which builds on work of Kazhdan and Lusztig [38], Ginzburg [11], and Lusztig [42, 43]. ${ }^{1}$

We now give an example that illustrates some of the points to be discussed in the sequel.

EXAMPLE 0.1. - Let $W$ be the dihedral group of order 8. It is generated by two elements $s, t$ which have order 2 and whose product has order 4 . Thus, $(W,\{s, t\})$ is a Coxeter system of type $B_{2}$. Let $\mathcal{H}$ be the corresponding Iwahori-Hecke algebra over the ring $A=\mathbb{Z}\left[u, u^{-1}\right]$, where $u$ is an indeterminate, see (1.1). Then $\mathcal{H}$ is an associative algebra with an identity $T_{1}$, generated by elements $T_{s}, T_{t}$ such that the following relations hold:

$$
T_{s}^{2}=u T_{1}+(u-1) T_{s}, \quad T_{t}^{2}=u T_{1}+(u-1) T_{t}, \quad T_{s} T_{t} T_{s} T_{t}=T_{t} T_{s} T_{t} T_{s} .
$$

Let $K$ be the field of fractions of $A$. Then the algebra $K \mathcal{H}=K \otimes_{A} \mathcal{H}$ is semisimple and, up to equivalence, its irreducible representations are given as follows (see $[12, \S 67 \mathrm{C}]$ ):

$$
\begin{array}{rrrrrr}
\text { ind: } & T_{s} \mapsto \quad u, & T_{t} \mapsto \quad u, & \varepsilon_{s}: & T_{s} \mapsto-1, T_{t} \mapsto u \\
\varepsilon: & T_{s} \mapsto-1, & T_{t} \mapsto-1, & \varepsilon_{t}: & T_{s} \mapsto \quad u, T_{t} \mapsto-1, \\
\rho: & T_{s} \mapsto\left[\begin{array}{rr}
-1 & 0 \\
2 & u
\end{array}\right], & T_{t} \mapsto\left[\begin{array}{rr}
u & u \\
0 & -1
\end{array}\right] .
\end{array}
$$

Let $A_{\mathfrak{p}}$ be the localization of $A$ in some non-zero prime ideal $\mathfrak{p}$, and let $k_{\mathfrak{p}}$ be its residue field. We consider the canonical map $A_{\mathfrak{p}} \rightarrow k_{\mathfrak{p}}$ and determine the corresponding decomposition matrix between the irreducible representations of the algebras $K \mathcal{H}$ and $k_{\mathfrak{p}} \mathcal{H}$. The 1-dimensional representations certainly remain irreducible; but they all become equal over $k_{\mathfrak{p}}$ precisely when $u+1 \in \mathfrak{p}$. Furthermore, the representation $\rho$ becomes reducible over $k_{\mathfrak{p}}$ if and only if the above two matrices have a common eigenvector. One easily checks that

\begin{tabular}{|c|c|c|c|c|c|c|c|}
\hline & \multicolumn{2}{|c|}{$(I)$} & \multicolumn{4}{|c|}{$(I I)$} & $(I I I)$ \\
\hline ind & 1 & 0 & 1 & 0 & 0 & 0 & 1 \\
\hline$\varepsilon_{s}$ & 1 & 0 & 0 & 1 & 0 & 0 & 1 \\
\hline$\varepsilon_{t}$ & 1 & 0 & 0 & 0 & 1 & 0 & 1 \\
\hline$\varepsilon$ & 1 & 0 & 0 & 0 & 0 & 1 & 1 \\
\hline$\rho$ & 0 & 1 & 1 & 0 & 0 & 1 & 2 \\
\hline
\end{tabular}
this happens if and only if $u^{2}+1 \in \mathfrak{p}$ or $2 \in \mathfrak{p}$. So, if the image of $u$ in $k_{\mathfrak{p}}$ is a root of unity then we find essentially three different cases where we obtain non-trivial decomposition matrices: (I) $u+1 \in \mathfrak{p}, 2 \notin \mathfrak{p} ;(\mathrm{II}) u^{2}+1 \in \mathfrak{p}, 2 \notin \mathfrak{p} ;$ (III) $u+1 \in \mathfrak{p}, 2 \in \mathfrak{p}$.

We may summarize these results as follows; let $\bar{u}$ be the image of $u$ in $k_{\mathrm{p}}$. Then we have: If $\operatorname{char}\left(k_{\mathfrak{p}}\right) \neq 2$, the decomposition matrix only depends on the order of $\bar{u}$ in $k_{\mathfrak{p}}^{\times}$. This is the prototype of the general picture; Conjecture 3.4 below will explain the special role of the prime 2 by the fact that this is the only prime dividing the order of $W$; see

\footnotetext{
${ }^{1}$ Note added June 1998. The generalization of this conjecture to the Dipper-James $q$-Schur algebra (see Leclerc and Thibon [40]) has been established recently by Varagnolo and Vasserot [55].
} 
Remark 3.6 for the case where $\bar{u}$ is not a root of unity in $k_{\mathrm{p}}$. Ariki's results actually apply also to Hecke algebras of type $B_{n}$; hence they yield an a priori proof for the matrices (I) and (II). Furthermore, the above matrices are parts of the $\ell$-modular decomposition matrices for the finite groups $\mathrm{SO}_{5}(q)$, where $\ell$ does not divide $q$.

Explicit results have been obtained in a number of cases; see [32, 34, 35, 19, 51] for classical types and [7, 25, 22, 49,50] for exceptional types (see also [15] for a survey). In the latter cases, these computations use in an essential way the computer algebra systems GAP [52] and MAPLE [10]. But they have also been a stimulus for theoretical research and a source of evidence-producing examples.

\section{HECKE ALGEBRAS AND $q$-SCHUR ALGEBRAS}

In this section, we describe the construction of Hecke algebras and $q$-Schur algebras, and explain how they appear in the representation theory of finite Chevalley groups.

1.1. Let $A$ be any commutative ring with 1 and $(W, S)$ a finite Coxeter system, i.e., $W$ is a finite group and $S$ is a subset of $W$ such that we have a presentation of the form

$$
\left.W=\langle s \in S| s^{2}=1 \text { and }(s t)^{m_{s t}}=1 \text { for } s, t \in S\right\rangle \quad \text { where } m_{s t}=\text { order of } s t .
$$

Let $\left(a_{s}\right)_{s \in S}$ be a collection of elements in $A$ such $a_{s}=a_{t}$ whenever $s, t \in S$ are conjugate in $W$. Then we let $H=H_{A}\left(W, S,\left(a_{s}\right)_{s \in S}\right)$ be the associative $A$-algebra with identity element $T_{1}$, defined by a presentation with generators $T_{s}(s \in W)$ and defining relations:

$$
T_{s}^{2}=a_{s} T_{1}+\left(a_{s}-1\right) T_{s} \quad(\text { for } s \in S), \quad \underbrace{T_{s} T_{t} T_{s} \cdots}_{m_{s t} \text { factors }}=\underbrace{T_{t} T_{s} T_{t} \cdots}_{m_{s t} \text { factors }} \quad \text { (for } s, t \in S) .
$$

We call $H$ the Iwahori-Hecke algebra associated with $(W, S)$ and with parameters $\left(a_{s}\right)_{s \in S}$. It is a non-trivial fact that $H$ has a basis $\left\{T_{w} \mid w \in W\right\}$, which is defined as follows. Any $w \in W$ can be expressed as $w=s_{1} \cdots s_{m}$ with $s_{i} \in S$. If $m$ is minimal possible, we call this a reduced expression and set $l(w):=m$. In this case, the element $T_{w}:=T_{s_{1}} \cdots T_{s_{m}}$ is independent of the choice of a reduced expression; see [6, Chap. IV, §2, Ex. 23]. Note that if $a_{s}=1$ for all $s \in S$ then $H$ is just the group algebra $A[W]$. We shall assume that all $a_{s}$ are units in $A$, which implies that all $T_{w}$ are invertible in $H$ and that $H$ is a symmetric algebra. (The latter property will not be used here explicitly, but see $[12, \S 68 \mathrm{C}]$.)

The above construction is functorial in the sense that if $f: A \rightarrow B$ is any homomorphism of commutative rings, we can regard $B$ as an $A$-module and then have a canonical isomorphism $B \otimes_{A} \mathcal{H} \cong H_{B}\left(W, S,\left(b_{s}\right)_{s \in S}\right)$ where $b_{s}=f\left(a_{s}\right)$ for all $s \in S$.

REMARK 1.2. - There is a generalization to the case of finite complex reflection groups, where the quadratic relations for the generators $T_{s}$ are replaced by some higher order polynomial relations. These are the cyclotomic Hecke algebras defined by Broué and Malle [8]; see also Ariki and Koike [3]. Note, however, that some properties are not yet 
established in full generality for them; for example, the existence of a basis over $A$ which is indexed by the elements of the underlying group and has good properties.

We now explain how Hecke algebras arise in the representation theory of finite Chevalley groups. We will only consider the simplest possible case as far as modular representations are concerned, and refer to [9, Chap. 10] and [24, §2] for further reading.

1.3. Assume that $W$ is the Weyl group of an (untwisted) finite Chevalley group $G$ defined over the finite field with $q$ elements. Consider the permutation module $A[G / B]$ on the cosets of a Borel subgroup $B \subseteq G$. Then Iwahori has shown that

$$
H_{A}\left(W, S,\left(a_{s}\right)_{s \in S}\right) \cong \operatorname{End}_{G}(A[G / B]), \quad \text { where } a_{s}=q \cdot 1_{A} \text { for all } s \in S,
$$

see [6, Chap. IV, $\S 2$, Ex. 22-24]. (If $G$ is a Chevalley group of twisted type, then $a_{s}$ may be some power of $q$; but note that not all possible choices of the parameters $\left(a_{s}\right)$ do arise.) The standard example is the case when $G=\mathrm{GL}_{n}(q), W=\mathfrak{S}_{n}$ (the symmetric group on $n$ letters), and $B$ is the subgroup of invertible upper triangular matrices.

Now we take $A$ to be a discrete valuation ring $\mathcal{O}$ of characteristic 0 , whose residue field $k$ has characteristic $\ell$. Assume that $\ell$ does not divide $q$, so that $q$ is an invertible element of $\mathcal{O}$. Let $F$ be the field of fractions of $\mathcal{O}$ and assume that $\mathcal{O}$ is chosen to be large enough so that $F[G]$ is split. We consider the usual $\ell$-modular decomposition matrices for the algebras $\mathcal{O}[G]$ and $\operatorname{End}_{G}(\mathcal{O}[G / B])$ (cf. (2.1) below).

THEOREM 1.4 (Dipper). - The decomposition matrix $D$ of the algebra $\operatorname{End}_{G}(\mathcal{O}[G / B])$ is a submatrix of the decomposition matrix of $\mathcal{O}[G]$. If $\ell$ does not divide $q-1$, then the embedding of the first matrix into the second is given as follows:

\begin{tabular}{|c|c|}
\hline$D$ & $*$ \\
\hline 0 & $*$ \\
\hline
\end{tabular}

where the rows are labelled by the simple $F[G]$-modules,

the columns are labelled by the simple $k[G]$-modules,

and the rows of $D$ correspond to the constituents of $F[G / B]$.

If $\ell$ does not divide $q-1$, this is proved as follows. Let $M$ be an indecomposable direct summand of $\mathcal{O}[G / B]$. Since $\ell$ does not divide the order of $B$ (which is $q^{m}(q-1)^{r}$ for suitable $m, r \geq 1$ ), the module $\mathcal{O}[G / B]$ is projective. So $M$ is in fact a projective indecomposable $\mathcal{O}[G]$-module. Now we extend scalars from $\mathcal{O}$ to $F$, and write $F N=$ $F \otimes_{\mathcal{O}} N$ for any $\mathcal{O}$-module $N$. Since $F[G]$ is semisimple, the module $F M$ splits up into a direct sum of simple $F[G]$-modules, and the multiplicities determine a column of the decomposition matrix of $\mathcal{O}[G]$, by Brauer's reciprocity law (see $[12, \S 18 \mathrm{~B}]$ ).

On the other hand, $M$ corresponds to a primitive idempotent of $\operatorname{End}_{G}(\mathcal{O}[G / B])$, and hence to a projective indecomposable module $P_{M}$ for this algebra. Now we note that extending scalars from $\mathcal{O}$ to $F$ induces an isomorphism $F \operatorname{End}_{G}(\mathcal{O}[G / B]) \cong \operatorname{End}_{G}(F[G / B])$. It follows that the splitting of $F M$ into a direct sum of simple $F[G]$-modules corresponds 
to a splitting of $F P_{M}$ into a direct sum of simple $\operatorname{End}_{G}(F[G / B])$-modules. The multiplicities are the same as before, and they also determine a column of the decomposition matrix of $\operatorname{End}_{G}(\mathcal{O}[G / B])$. - The general case is based on the fact that there exists an exact sequence $0 \rightarrow N \rightarrow P \rightarrow \mathcal{O}[G / B] \rightarrow 0$ of $\mathcal{O}[G]$-modules such that $P$ is projective and $F N$ and $F[G / B]$ have no irreducible constituent in common; for details see [14, $\S 2]$.

1.5. Let $G=\mathrm{GL}_{n}(q)$. Then Dipper and James have constructed an enlargement of the Hecke algebra which eventually yields the whole decomposition matrix of $G$. For this purpose, consider the symmetric group $\mathfrak{S}_{n}$, regarded as a Coxeter group on the generators $\left\{s_{1}, \ldots, s_{n-1}\right\}$, where $s_{i}=(i, i+1)$ for all $i$. Let $H_{n}$ be the corresponding Iwahori-Hecke algebra over a commutative ring $A$ and with parameter $u$. (Note that all generators $s_{i}$ are conjugate in $\mathfrak{S}_{n}$.) For each partition $\lambda \vdash n$, we have a corresponding Young subgroup $\mathfrak{S}_{\lambda} \subseteq \mathfrak{S}_{n}$; if $\lambda$ has parts $\lambda_{1}, \lambda_{2}, \ldots$ we have $\mathfrak{S}_{\lambda} \cong \mathfrak{S}_{\lambda_{1}} \times \mathfrak{S}_{\lambda_{2}} \times \ldots$. Let $H_{\lambda}$ be the subalgebra of $H_{n}$ generated by the elements $T_{w}$ for $w \in \mathfrak{S}_{\lambda}$, and let ind ${ }_{\lambda}: H_{\lambda} \rightarrow A$ be the representation given by $\operatorname{ind}_{\lambda}\left(T_{w}\right)=u^{l(w)}$. Then the module $M_{\lambda}:=H_{n} \otimes_{H_{\lambda}} \operatorname{ind}_{\lambda}$ is a Hecke algebra analogue of the permutation module of $\mathfrak{S}_{n}$ on the cosets of $\mathfrak{S}_{\lambda}$. We set

$$
\mathcal{S}_{A}(n, u):=\operatorname{End}_{H_{n}}\left(\bigoplus_{\lambda \vdash n} M_{\lambda}\right) .
$$

This is the Dipper-James $q$-Schur algebra, defined and studied in $[17,18]$. We have

$$
\mathcal{S}_{\mathcal{O}}(n, q) \cong \operatorname{End}_{G}\left(\bigoplus_{\lambda \vdash n} \mathcal{O}\left[G / P_{\lambda}\right]\right), \quad \text { see [17, Theorem 2.24], }
$$

where $\mathcal{O}$ is a discrete valuation ring as above and $P_{\lambda} \subseteq G$ denotes the parabolic subgroup of all invertible block upper triangular matrices with blocks given by the parts of $\lambda$.

Theorem 1.6 (Dipper and James $[17, \S 6]$ ). - The decomposition matrix of $\mathcal{S}_{\mathcal{O}}(n, q)$ is a part of the decomposition matrix of $\mathcal{O}[G]$. Moreover, the latter matrix can be calculated from the decomposition matrices of those algebras $\mathcal{S}_{\mathcal{O}}\left(r, q^{d}\right)$ for which $d r \leq n$.

There are also $q$-Schur algebras associated with other types of finite Coxeter groups. The most far-reaching such generalizations are due to Gruber and Hiss [29], where a result similar to Theorem 1.6 for finite classical groups and so-called linear primes $\ell$ is obtained.

\section{A GENERAL SETTING FOR DECOMPOSITION MAPS}

Let $A$ be any integral domain and $H$ be an associative $A$-algebra, finitely generated and free over $A$. (All of our rings and algebras will have identity elements, and ring homomorphisms will respect them.) Let $K$ be the field of fractions of $A$. By extension of scalars, we obtain a $K$-algebra $K H$. (If $A \rightarrow B$ is any homomorphism into a commutative ring $B$ and $M$ is any $A$-module, we regard $B$ as an $A$-module and set $B M:=B \otimes_{A} M$.)

Let $\mathfrak{p} \subset A$ be a non-zero prime ideal. We consider the corresponding localized ring $A_{\mathfrak{p}}$, and denote its residue field by $k_{\mathfrak{p}}$. We wish to describe a setting in which the canonical map 
$A_{\mathfrak{p}} \rightarrow k_{\mathfrak{p}}$ induces a homomorphism $d_{\mathfrak{p}}: R_{0}(K H) \rightarrow R_{0}\left(k_{\mathfrak{p}} H\right)$ between the Grothendieck groups $^{2}$ of $K H$ and $k_{\mathfrak{p}} H$, which we will then call the $\mathfrak{p}$-modular decomposition map of $H$. The corresponding decomposition matrix will be the matrix of $d_{\mathfrak{p}}$ with respect to the standard bases of $R_{0}(K H)$ and $R_{0}\left(k_{\mathfrak{p}} H\right)$ consisting of the classes of simple modules.

2.1. The "usual" case of discrete valuation rings. If $A_{\mathfrak{p}}$ is a discrete valuation ring, we can define the desired decomposition map as follows: for a given $K H$-module $V$, there exists a $K$-basis such that the $A_{\mathfrak{p}}$-submodule $M \subset V$ spanned by that basis is invariant under the action of $H$; then $M / \mathfrak{p} M$ can be regarded as a $k_{\mathfrak{p}} H$-module, and we define $d_{\mathfrak{p}}$ by sending the class of $V$ to the class of $M / \mathfrak{p} M$. For the details of this construction, see $[12, \S 16 \mathrm{C}]$. The assumption that $A_{\mathfrak{p}}$ is a discrete valuation ring is satisfied if and only if $A_{\mathfrak{p}}$ is Noetherian of dimension 1 and integrally closed in $K$ (see [47, Theorem 11.2]). For example, this holds if $A$ is the ring of integers in some algebraic number field. This is the case which is usually considered in the modular representation theory of finite groups.

For applications to Hecke algebras, we have to deal with more general types of rings.

2.2. We consider the following set-up. First, in order to avoid technical complications, we will assume that $A$ has been chosen large enough so that the algebra $k_{\mathfrak{p}} H$ is split, i.e., the endomorphism ring of any simple module consists only of the scalar multiples of the identity. (This is no serious condition since every finite-dimensional algebra becomes split after a finite field extension.)

Next, assume that there exists some discrete valuation ring $\mathcal{O} \subseteq K$ with maximal ideal $J(\mathcal{O})$ such that $A \subseteq \mathcal{O}$ and $J(\mathcal{O}) \cap A=\mathfrak{p}$. (This condition is more serious, but it is satisfied, for example, whenever $A_{\mathfrak{p}}$ is a regular local ring, see [47, Ex. 14.4]; this is sufficient for all our applications. One could avoid that condition by working with general valuation rings.) Let $k$ be the residue field of $\mathcal{O}$; we may regard $k$ as an extension of $k_{\mathrm{p}}$. Applying the discussion in (2.1) with $A_{\mathfrak{p}}$ replaced by $\mathcal{O}$, we obtain a decomposition map $d_{J(\mathcal{O})}: R_{0}(K H) \rightarrow R_{0}(k H)$. Since $k_{\mathrm{p}} H$ is assumed to be split, the scalar extension from $k_{\mathfrak{p}}$ to $k$ defines an isomorphism $R_{0}\left(k_{\mathfrak{p}} H\right) \cong R_{0}(k H)$ which preserves the classes of simple modules. Identifying these two Grothendieck groups, we obtain a decomposition map

$$
d_{J(\mathcal{O})}: R_{0}(K H) \rightarrow R_{0}\left(k_{\mathfrak{p}} H\right)
$$

A priori, this map depends on the choice of $\mathcal{O}$. But we have, see $[27, \S 2]$ :

Proposition 2.3 (Uniqueness of decomposition maps). - Assume that $A$ is integrally closed in $K$, and recall that $k_{\mathrm{p}} H$ is assumed to be split. Then, in the above setting, the map $d_{J(\mathcal{O})}: R_{0}(K H) \rightarrow R_{0}\left(k_{\mathfrak{p}} H\right)$ does not depend on the choice of $\mathcal{O}$.

\footnotetext{
${ }^{2}$ Recall that $R_{0}(K H)$ is the abelian group generated by expressions [V], one for each finite dimensional $K H$-module $V$, subject to relations $[V]=\left[V^{\prime}\right]+\left[V^{\prime \prime}\right]$ for each short exact sequence $0 \rightarrow V^{\prime} \rightarrow V \rightarrow$ $V^{\prime \prime} \rightarrow 0$ of $K H$-modules. The group $R_{0}(K H)$ is free abelian, with a basis $\{[S] \mid S$ simple $\}$.
} 
It will be useful to indicate briefly how this is proved. Let $R_{0}^{+}(K H)$ be the submonoid of $R_{0}(K H)$ consisting of the classes of $K H$-modules. Let $X$ be an indeterminate over $K$, and $\operatorname{Maps}(H, K[X])$ be the monoid of all maps from $H$ to $K[X]$ (with pointwise defined multiplication). We define a monoid homomorphism $\mathfrak{x}_{K}: R_{0}^{+}(K H) \rightarrow \operatorname{Maps}(H, K[X])$ by associating with the class of a $K H$-module $V$ the map which assigns to an element $h \in H$ the characteristic polynomial of $1 \otimes h \in K H$ in its action on $V$. In a similar way, we also define a monoid homomorphism $\mathfrak{x}_{k_{\mathfrak{p}}}: R_{0}^{+}\left(k_{\mathfrak{p}} H\right) \rightarrow \operatorname{Maps}\left(H, k_{\mathfrak{p}}[X]\right)$.

Since $A$ is integrally closed in $K$, the image of $\mathfrak{x}_{K}$ already lies in $\operatorname{Maps}(H, A[X])$ (see $\left[27\right.$, Lemma 2.10]). Now we have a canonical map $t_{\mathfrak{p}}: \operatorname{Maps}(H, A[X]) \rightarrow \operatorname{Maps}\left(H, k_{\mathfrak{p}}[X]\right)$, given by applying $A_{\mathfrak{p}} \rightarrow k_{\mathfrak{p}}$ to the coefficients of polynomials. Using the construction of $d_{J(\mathcal{O})}$ we easily check that the following diagram is commutative:

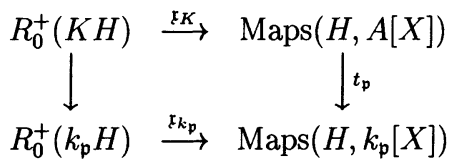

where the left hand vertical arrow is given by $d_{J(\mathcal{O})}$. Finally, the assumption that $k_{\mathrm{p}} H$ is split implies that the Brauer-Nesbitt Lemma holds, i.e., the map $\mathfrak{x}_{k_{\mathfrak{p}}}$ is injective (see [27, Prop. 2.5]). So there is at most one map $R_{0}^{+}(K H) \rightarrow R_{0}^{+}\left(k_{\mathrm{p}} H\right)$ making (D) commutative. In particular, Proposition 2.3 is proved, and we have a well-defined decomposition map

$$
d_{\mathfrak{p}}: R_{0}(K H) \rightarrow R_{0}\left(k_{\mathfrak{p}} H\right) .
$$

(This characterisation of decomposition maps appears in [27, Prop. 2.11]. Note, however, that the right hand vertical arrow in the diagram in [loc. cit.] has to be defined as above.)

The following result gives a condition for a decomposition map to be "trivial".

Theorem 2.4 (Tits' Deformation Theorem). - In the above set-up, assume that $K H$ is also split and that the radicals of $K H$ and $k_{\mathfrak{p}} H$ have the same dimension. Then $d_{\mathfrak{p}}$ is an isomorphism which preserves the classes of simple modules.

This is proved in $[12, \S 68 \mathrm{~A}]$ under the assumption that $K H$ and $k_{\mathrm{p}} H$ are semisimple. In order to reduce to this case, we argue as follows. Let $\mathcal{O} \subseteq K$ be a discrete valuation ring as in (2.2) with residue field $k \supseteq k_{\mathfrak{p}}$, and let $J \subseteq \mathcal{O} H$ be the intersection of the radical of $K H$ with $\mathcal{O} H$. Then $J$ is a pure submodule of $\mathcal{O} H$ and we set $\bar{H}:=\mathcal{O} H / J$. Since the radical acts as 0 on every simple module, we can identify $R_{0}(K H)=R_{0}(K \bar{H})$. On the other hand, since $J$ is nilpotent, $k J$ is contained in the radical of $k H$. But we have in fact equality, thanks to our assumptions. (Note that the radicals of $k_{\mathfrak{p}} H$ and $k H$ have the same dimension, since $k_{\mathrm{p}} H$ is split.) Hence, using the isomorphism $k H / k J \cong k \bar{H}$, we can also identify $R_{0}(k H)=R_{0}(k \bar{H})$, and it remains to apply [12, Cor. 68.20].

2.5. We apply the above discussion to obtain a factorization result for decomposition maps (cf. [27, Prop. 2.5]). For this purpose, assume that $A$ is a Noetherian ring with $\operatorname{dim} A=2$ and that $A$ is integrally closed in $K$. 
Let $\mathfrak{p}, \mathfrak{q}$ be prime ideals of $A$ such that $\{0\} \neq \mathfrak{q} \subset \mathfrak{p}$. Then $\mathfrak{q}$ has height 1 and $\operatorname{dim} A_{\mathfrak{q}}=1$. So, by (2.1), we have a well-defined decomposition map $d_{\mathfrak{q}}: R_{0}(K H) \rightarrow R_{0}\left(k_{\mathfrak{q}} H\right)$. Assume also that $A / \mathfrak{q}$ is integrally closed in its field of fractions $k_{\mathfrak{q}}$. Then, since $\operatorname{dim} A / \mathfrak{q}=1$, the localization of $A / \mathfrak{q}$ in the prime ideal $\mathfrak{p} / \mathfrak{q}$ is a discrete valuation ring and so, again by $(2.1)$, the canonical map $A / \mathfrak{q} \rightarrow k_{\mathfrak{p}}$ induces a decomposition map $d_{\mathfrak{p}}^{\mathfrak{q}}: R_{0}\left(k_{\mathfrak{q}} H\right) \rightarrow R_{0}\left(k_{\mathfrak{p}} H\right)$.

Proposition 2.6 (Factorization of decomposition maps). - Let $\{0\} \neq \mathfrak{q} \subset \mathfrak{p} \subset A$ be as in (2.5), and assume also that $k_{\mathfrak{p}} H$ is split. Then we have a decomposition map $d_{\mathfrak{p}}$ as in Proposition 2.3 and the following diagram is commutative:

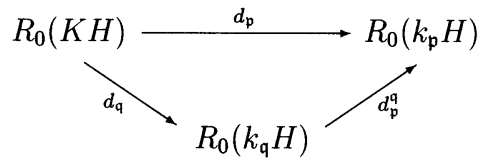

Indeed, the assumption on $A / \mathfrak{q}$ implies that the image of $\mathfrak{x}_{\mathfrak{q}}$ lies in $\operatorname{Maps}(H, A / \mathfrak{q}[X])$. So the composition $d_{\mathfrak{p}}^{\mathfrak{q}} \circ d_{\mathfrak{q}}$ defines a map from $R_{0}^{+}(K H)$ to $R_{0}^{+}\left(k_{\mathfrak{p}} H\right)$ which makes the diagram (D) commutative. Hence that composition must be equal to $d_{\mathfrak{p}}$.

2.7. Let $A$ be as in (2.5). We now fix a height 1 prime ideal $\mathfrak{q}$ such that $A / \mathfrak{q}$ is integrally closed in $k_{\mathfrak{q}}$ and $k_{\mathfrak{q}} H$ is split. Let $P(\mathfrak{q})$ be the set of all maximal ideals of $A$ which contain $\mathfrak{q}$. We close this section with a result which shows that the decomposition maps $d_{\mathfrak{p}}$, for $\mathfrak{p} \in P(\mathfrak{q})$, are "generically" determined by $d_{\mathfrak{q}}$. In fact, by Proposition 2.6, we have a factorization $d_{\mathfrak{p}}=d_{\mathfrak{p}}^{\mathfrak{q}} \circ d_{\mathfrak{q}}$ for all $\mathfrak{p} \in P(\mathfrak{q})$ such that $k_{\mathfrak{p}} H$ is split. We claim that

$k_{\mathfrak{p}} H$ is split and $d_{\mathfrak{p}}^{\mathfrak{q}}$ is trivial for all but finitely many $\mathfrak{p} \in P(\mathfrak{q})$.

(Here, "trivial" means an isomorphism which preserves the classes of simple modules.)

This is proved as follows (cf. the argument given in [21, Prop. 5.5]). To simplify notation, let $R=A / \mathfrak{q}$ and $F=k_{\mathfrak{q}}$; note that $R$ is a Dedekind domain. By Wedderburn's Theorem, there exists a surjective homomorphism of $F$-algebras $\psi: F H \rightarrow \bigoplus_{i=1}^{n} M_{n_{i}}(F)$, with $\operatorname{ker}(\psi)$ nilpotent, where $M_{l}(F)$ denotes the full matrix algebra of degree $l$ over $F$.

Now take a basis $B$ of $F H$ which consists of a basis of $\operatorname{ker}(\psi)$ and a set of elements which are mapped to an $R$-basis of $\bigoplus_{i=1}^{n} M_{n_{i}}(R)$. We also fix an $R$-basis of $R H$ and express each $b \in B$ as a linear combination of that basis, where the coefficients are quotients of elements of $R$. Taking the product of all denominators in all coefficients of all basis elements $b \in B$, we see that there exists some $0 \neq d \in R$ such that $d b \in R H$ for all $b \in B$. Then we have in fact $\psi(R[1 / d] H)=\bigoplus_{i=1}^{n} M_{n_{i}}(R[1 / d])$.

Now let $\mathfrak{p} \in P(\mathfrak{q})$ be such that $d \notin \mathfrak{p} / \mathfrak{q}$; note that only finitely many $\mathfrak{p}$ 's do not satisfy this condition. Then the quotient map $R=A / \mathfrak{q} \rightarrow k_{\mathfrak{p}}$ extends to a map $R[1 / d] \rightarrow k_{\mathfrak{p}}$, and extension of scalars along this map gives rise to a surjective homomorphism of $k_{\mathfrak{p}}$-algebras $\psi_{\mathfrak{p}}: k_{\mathfrak{p}} H \rightarrow \bigoplus_{i=1}^{n} M_{n_{i}}\left(k_{\mathfrak{p}}\right)$, with $\operatorname{ker}\left(\psi_{\mathfrak{p}}\right)$ nilpotent. We conclude that $k_{\mathfrak{p}} H$ is split and that $\operatorname{ker}\left(\psi_{\mathfrak{p}}\right)$ is the radical of $k_{\mathfrak{p}} H$, having the same dimension as the radical of $F H$. Now $d_{\mathfrak{p}}^{\mathfrak{q}}$ is induced by $R[1 / d] \rightarrow k_{\mathfrak{p}}$, and it remains to use Tits' Deformation Theorem 2.4. 


\section{DECOMPOSITION MAPS AND ROOTS OF UNITY}

We now fix a special choice of the ring $A$. Let $F$ be an algebraic number field and $R \subset F$ be a subring which is a Dedekind ring with field of fractions $F$. (Examples: $R$ is the ring of all algebraic integers in $F$ or $R$ is a discrete valuation ring in $F$.) Let $A=R\left[v, v^{-1}\right]$ be the ring of Laurent polynomials over $R$ in an indeterminate $v$ and $K$ be its field of fractions. Let $(W, S)$ be a finite Coxeter system, and consider the following generic Iwahori-Hecke algebra:

$$
\mathcal{H}:=H_{A}\left(W, S,\left(a_{s}\right)_{s \in S}\right), \quad \text { where } a_{s}=v^{2 c_{s}} \text { with } 0 \neq c_{s} \in \mathbb{N} \text { for all } s \in S .
$$

(It would also be possible to work with a $q$-Schur algebra or with a cyclotomic algebra; but let us stick to this specific case.) We will assume throughout that $R$ is large enough; in particular, $R$ will contain all the square roots and roots of unity that we will need below and the group algebra $F[W]$ will be split semisimple. The fact that the parameters $a_{s}$ have square roots implies that the algebra $K \mathcal{H}$ is split semisimple (see $[1,5,41]$ ).

Our aim is to understand the decomposition maps for $\mathcal{H}$ associated with the various prime ideals in $A$. First note that our ring $A$ satisfies all the conditions of Section 2: since $R$ is a Dedekind ring, the localization of $A$ in any prime ideal $\mathfrak{p}$ is a regular local ring; this implies, in particular, that $A$ is integrally closed in its field of fractions $K$. (For these facts, see $[47, \S 19]$.) Moreover, there always exists a discrete valuation ring $\mathcal{O}$ as required in Proposition 2.3 (see [47, Ex. 14.4]). Note also that $\operatorname{dim} A=2$.

3.1. We consider the following data: an invertible element $\zeta \in R$ and a rational prime number $\ell$ which is not invertible in $R$. Let $\mathfrak{q} \subset A$ be the kernel of the map $A \rightarrow R$, $v \mapsto \zeta$, and $\mathfrak{p} \subset A$ be any maximal ideal containing $\mathfrak{q}$, whose residue field $k_{\mathfrak{p}}$ is finite of characteristic $\ell$. Note that $A / \mathfrak{q}=R$ is integrally closed in its field of fractions, which is $k_{\mathfrak{q}}=F$. Enlarging $R$ if necessary, we can assume that $k_{\mathfrak{p}} \mathcal{H}$ is split. Then all conditions of Proposition 2.6 are satisfied, and so we have a factorization of decomposition maps: $d_{\mathfrak{p}}=d_{\mathfrak{p}}^{\mathfrak{q}} \circ d_{\mathfrak{q}}$. Thus, we have the following picture:

- The map $d_{\mathfrak{q}}$ between the Grothendieck groups of $K \mathcal{H}$ and $F \mathcal{H}$ is induced by the specialization $A \rightarrow R, v \mapsto \zeta$; we simply denote it $d_{\zeta}$.

- The map $d_{\mathfrak{p}}^{\mathfrak{q}}$ is induced by the canonical map $R_{\overline{\mathfrak{p}}} \rightarrow k_{\mathfrak{p}}$, where $\overline{\mathfrak{p}}$ is the image of $\mathfrak{p}$ in $R$; we will denote it $d_{\ell}^{\zeta}$.

So, our factorization reads $d_{\mathfrak{p}}=d_{\ell}^{\zeta} \circ d_{\zeta}$. We now give a number of examples which illustrate the above set-up. Note that by a general semisimplicity criterion (see [30] or [27]), the algebra $F \mathcal{H}$ is semisimple unless $\zeta$ is a root of unity.

EXAMPLE 3.2. - Let $\mathfrak{p} \subset A$ be a maximal ideal such that $k_{\mathfrak{p}}$ has characteristic $\ell>0$ and the image of $v$ in $k_{\mathfrak{p}}$ is 1 . Then $k_{\mathfrak{p}} \mathcal{H}$ is nothing but the group algebra of $W$ over $k_{\mathfrak{p}}$.

For $r \geq 0$ let $\zeta_{r} \in R$ be a root of unity such that $\zeta_{r}^{\ell^{r}}=1$. Then, regardless of what is $r$, the image of $\zeta_{r}$ in $k_{\mathfrak{p}}$ is 1 . Hence, if we let $\mathfrak{q}_{r} \subset A$ be the kernel of the canonical 
map $A \rightarrow R, v \mapsto \zeta_{r}$, then $\mathfrak{q}_{r} \subset \mathfrak{p}$ and we are in the set-up of (3.1). Note that, in the corresponding factorization $d_{\mathfrak{p}}=d_{\ell}^{\zeta_{r}} \circ d_{\zeta_{r}}$, the two maps on the right hand side depend on $r$ but the left hand side does not. Hence, taking once $r=0$ and then $r \geq 1$, we obtain:

$$
d_{\ell}^{1} \circ d_{1}=d_{\ell}^{\zeta_{r}} \circ d_{\zeta_{r}} \quad \text { for all } r \geq 1 .
$$

We can interprete this as follows. Since $\zeta_{0}=1$, we have $F \mathcal{H}=F[W]$. Hence $d_{\ell}^{1}$ is just the $\ell$-modular decomposition map for $W$. Since $F[W]$ is split semisimple, Tits' Deformation Theorem 2.4 implies that $d_{1}$ is an isomorphism which preserves the classes of simple modules. Thus, the left hand side of the above identity may be regarded as a lift of $d_{\ell}^{1}$ to $\mathcal{H}$. Hence the maps $d_{\zeta_{r}}(r \geq 1)$ may be viewed as approximations to $d_{\ell}^{1}{ }^{3}$

EXAMPLE 3.3. - Let $W$ be the Weyl group of a finite Chevalley group $G$ over a field with $q$ elements, as in (1.3). Assume that $q$ is invertible in $R$ and $F[G]$ is split. Then we have $R \mathcal{H} \cong \operatorname{End}_{G}(R[G / B])$ under the specialization $v \mapsto \sqrt{q}$. Let $\mathfrak{p} \subset A$ be a maximal ideal such that $k_{\mathfrak{p}}$ has characteristic $\ell>0$ and such that $v-\sqrt{q} \in \mathfrak{p}$. Let $d \geq 1$ be the multiplicative order of $q$ modulo $\ell$; in particular, $d$ divides $\ell-1$. Since $R$ is large enough, we can find a primitive $2 d$-th root of unity $\zeta \in R$. This can be chosen so that $\sqrt{q}$ and $\zeta$ have the same image in $k_{\mathfrak{p}}$, and so we have $v-\zeta \in \mathfrak{p}$. Hence, each of $v-\sqrt{q}$ and $v-\zeta$ generate height 1 prime ideals which are contained in $\mathfrak{p}$ and such that we are in the set-up of (3.1). This yields an identity:

$$
d_{\ell}^{\sqrt{q}} \circ d_{\sqrt{q}}=d_{\ell}^{\zeta} \circ d_{\zeta}
$$

Now the left hand side has the following interpretation. The map $d_{\ell}^{\sqrt{q}}$ is the decomposition map which determines a part of the $\ell$-modular decomposition matrix of $G$ as in Proposition 1.4. But, since $F$ has characteristic 0 , the algebra $F \mathcal{H} \cong \operatorname{End}_{G}(F[G / B])$ is semisimple and so, by Tits' Deformation Theorem $2.4, d_{\sqrt{q}}$ is an isomorphism which preserves the classes of simple modules. Thus, the left hand side may be regarded as a lift of $d_{\ell}^{\sqrt{q}}$ to $\mathcal{H}$, and the map $d_{\zeta}$ is an approximation to it.

The above examples are taken from [21]. Motivated by the results of explicitly workedout examples - as mentioned in the introduction - we can now state:

ConjeCture 3.4. - In the set-up of (3.1), assume that we have $\zeta^{\ell-1}= \pm 1$ and that $\ell$ is coprime to the order of $W$ and to all $c_{s}(s \in S)$. Then the decomposition map $d_{\ell}^{\zeta}: R_{0}(F \mathcal{H}) \rightarrow R_{0}\left(k_{\mathfrak{p}} \mathcal{H}\right)$ is an isomorphism which preserves the classes of simple modules.

The condition $\zeta^{\ell-1}= \pm 1$ means that the image of $\zeta^{2}$ in $k_{\mathfrak{p}}$ lies in the prime field $\mathbb{F}_{\ell}$; hence so do the parameters of $k_{\mathrm{p}} \mathcal{H}$.

In the framework of Example 3.3, this conjecture says that if $\ell$ does not divide the order of $W$, then the part of the $\ell$-decomposition matrix of $G$ which is determined by the Hecke algebra $\mathcal{H}$ only depends on the order of $q$ modulo $\ell$, but not on $q$ and $\ell$ themselves.

\footnotetext{
${ }^{3} \mathrm{R}$. Rouquier once conjectured that $d_{\ell}^{1}$ might be determined by the maps $d_{\zeta_{r}}(r \geq 1)$, but he found a counter-example for $W=\mathfrak{S}_{13}$ and $\ell=2$.
} 
REMARK 3.5. - The above conjecture has first been stated by James in [32, §4], for Iwahori-Hecke algebras and $q$-Schur algebras associated with the symmetric group $\mathfrak{S}_{n}$. The general form is taken from [21, (5.6)]. Actually, James even gives a weaker condition on $\ell$, namely that $d \ell>n$ where $d \geq 1$ is the order of $\zeta^{2}$. This can also be generalized to other types (see the hypothesis of [27, Theorem 5.4] for such a generalization). We will not need to go into this here: the whole point of any form of such conditions is that it yields a bound for $\ell$ which is explicitly computable in terms of $(W, S)$. Note that, by the discussion in (2.7), the conclusion of the above conjecture can be seen to hold for all but finitely many primes $\ell$. Thus, the situation is reminiscent of that in the representation theory of simple algebraic groups; see [53].

In addition to the examples mentioned in the introduction, the conjecture is known to hold in the following situation. Assume that $W$ is a finite Weyl group and that $c_{s}=1$ for all $s \in S$. Let $P_{W}=\sum_{w \in W} v^{2 l(w)}$ be the Poincaré polynomial of $W$. Then the conjecture holds if $\zeta \in R$ is a simple root of $P_{W}$. The proof uses the Brauer-Dade theory of blocks with cyclic defect groups (applied to a finite Chevalley group with Weyl group $W$ ) and an adaptation of this theory to Iwahori-Hecke algebras; see [21].

REMARK 3.6. - In all of the above considerations, $\mathfrak{q}$ is a height 1 prime ideal so that $k_{\mathfrak{q}}$ has characteristic 0 . One may ask what happens if $k_{\mathrm{q}}$ has characteristic $\ell>0$ in which case $v$ necessarily maps to an indeterminate over $k_{\mathbf{q}}$. Then, by Fleischmann [20], the map $d_{\mathfrak{q}}$ is an isomorphism which preserves the classes of simple modules, unless $\ell$ is very small (i.e., a so-called "bad" prime for $W$ ). Furthermore, Gyoja [31] has proved that if $c_{s}=1$ for all $s \in S$, then $d_{\mathfrak{q}}$ has an interesting interpretation in terms of the multiplicities of the irreducible representations of $W$ in the Kazhdan-Lusztig cell representations of $W$.

REMARK 3.7. - Let $A$ be any Noetherian, integrally closed, integral domain, and $H$ an $A$-algebra, finitely generated and free over $A$. Assume that $\operatorname{dim} A=2$, and let $\mathfrak{q} \subset \mathfrak{p}$ be prime ideals with $\mathfrak{q}$ of height $1, \mathfrak{p}$ maximal, and $A / \mathfrak{q}$ integrally closed. One might ask if it is possible to generalize Conjecture 3.4 to this situation. We impose the condition:

Assume that $k_{\mathfrak{q}^{\prime}} H$ is semisimple for every height 1 prime ideal $\mathfrak{q}^{\prime} \subset A$ with $\mathfrak{q} \neq \mathfrak{q}^{\prime} \subset \mathfrak{p}$. In $[27,(3.3)]$ it is shown for Iwahori-Hecke algebras that then, at least, the Grothendieck groups of $k_{\mathfrak{q}} H$ and $k_{\mathfrak{p}} H$ have the same rank. Moreover, the above semisimplicity conditions translate to the condition on $\ell$ in Conjecture 3.4.

In any case, the decomposition maps $d_{\zeta}: R_{0}(K \mathcal{H}) \rightarrow R_{0}(F \mathcal{H})$, where $\zeta \in R$ is a root of unity, are of central importance. However, these are not yet well-understood. For example, the proofs of the following results which are given in [21, Prop. 5.4 and Prop. 7.4] proceed by a reduction to related statements about finite groups (via Dipper's Theorem 1.4).

Proposition 3.8. - Assume that $W$ is a finite Weyl group and that $c_{s}=1$ for $s \in S$. Let $\zeta \in R$ be a root of unity of order $2 d$ and consider the specialization $A \rightarrow R, v \mapsto \zeta$. 
(a) The $\operatorname{map}_{\zeta}: R_{0}(K \mathcal{H}) \rightarrow R_{0}(F \mathcal{H})$ is surjective and $F_{0} \mathcal{H}$ is split, where $F_{0}=\mathbb{Q}[\zeta]$.

(b) Let $V, V^{\prime}$ be simple $K \mathcal{H}$-modules and $D_{V}, D_{V^{\prime}} \in \mathbb{Q}[v]$ be their generic degrees, as defined in $[5]$. If $d_{\zeta}([V])$ and $d_{\zeta}\left(\left[V^{\prime}\right]\right)$ are not disjoint, then $D_{V}$ and $D_{V^{\prime}}$ are divisible by the same power of the $2 d$-th cyclotomic polynomial in $v$.

It would be desirable to prove this entirely in the framework of Iwahori-Hecke algebras. ${ }^{4}$

\section{THE LASCOUX-LECLERC-THIBON CONJECTURE}

We shall now consider decomposition maps for generic algebras whose parameter is specialized to a root of unity over $\mathbb{Q}$. So we work with the ring $A=\mathbb{C}\left[v, v^{-1}\right]$ (where $v$ is an indeterminate) and a prime ideal $\mathfrak{p} \subset A$ which is generated by $v-\zeta$, where $\zeta \in \mathbb{C}$ is a primitive $2 d$-th root of unity $(d \geq 1)$. Let $\mathcal{H}_{n}$ be the generic Iwahori-Hecke algebra over $A$ associated with the symmetric group $\mathfrak{S}_{n}$ (where the parameters are $a_{s}=v^{2}$ for $s \in S$ ). Then the canonical map $A_{\mathfrak{p}} \rightarrow k_{\mathfrak{p}}=\mathbb{C}$ induces a decomposition map

$$
d_{\zeta}: R_{0}\left(K \mathcal{H}_{n}\right) \rightarrow R_{0}\left(\mathbb{C H}_{n}\right), \quad \text { where } K=\text { field of fractions of } A .
$$

Note that the notation is such that for $s \in S$ we have $T_{s}^{2}=\zeta^{2} T_{1}+\left(\zeta^{2}-1\right) T_{s}$ in $\mathbb{C H}_{n}$.

4.1. The simple $\mathbb{C}\left[\mathfrak{S}_{n}\right]$-modules are naturally parametrized by the partitions $\lambda \vdash n$ (see [33]). Hence so are the simple $K \mathcal{H}_{n}$-modules (using the specialization $v \mapsto 1$ and Tits' Deformation Theorem as in Example 3.2). Dipper and James [16] have shown (see [26, (4.2)] for a different proof) that the simple $\mathbb{C H}_{n}$-modules are parametrized by the $d$ regular partitions $\mu \vdash n$, that is, those partitions which do not have $d$ parts which are equal. Moreover, the decomposition matrix $D_{n}^{\zeta}=\left(d_{\lambda \mu}\right)$ associated with $d_{\zeta}$ has the shape

$$
D_{n}^{\zeta}=\left[\begin{array}{cccc}
1 & 0 & \cdots & 0 \\
* & 1 & \ddots & \vdots \\
& & \ddots & 0 \\
\vdots & & & 1 \\
& & & \\
* & \cdots & *
\end{array}\right]
$$

for some suitable ordering of the rows and columns. For any $n \geq 1$, let $\mathcal{F}_{n}$ be a $\mathbb{C}$ vectorspace with a basis $\{[\lambda] \mid \lambda \vdash n\}$. Let $\mathcal{K}_{n} \subseteq \mathcal{F}_{n}$ be the subspace generated by all elements $P_{\mu}:=\sum_{\lambda} d_{\lambda \mu}[\lambda]$ where $\mu \vdash n$ is $d$-regular. Then $\mathcal{K}_{n}$ and $\mathcal{F}_{n}$ may be identified with the dual spaces of $\mathbb{C} \otimes_{\mathbb{Z}} R_{0}\left(\mathbb{C H}_{n}\right)$ and $\mathbb{C} \otimes_{\mathbb{Z}} R_{0}\left(K \mathcal{H}_{n}\right)$, respectively, and the embedding $\mathcal{K}_{n} \subseteq \mathcal{F}_{n}$ is the transpose of $d_{\zeta}$. Thus, the bases $\left\{P_{\mu}\right\}$ and $\{[\lambda]\}$ of $\mathcal{K}_{n}$ and $\mathcal{F}_{n}$, respectively, are dual to the standard bases of $\mathbb{C} \otimes_{\mathbb{Z}} R_{0}\left(\mathbb{C H}_{n}\right)$ and $\mathbb{C} \otimes_{\mathbb{Z}} R_{0}\left(K \mathcal{H}_{n}\right)$ given by the simple modules. We extend this to $n=0$, where $\mathcal{F}_{0}=\mathcal{K}_{0}=\mathbb{C}[\emptyset]$ and $\emptyset$ is the empty partition.

\footnotetext{
${ }^{4}$ Note added June 1998. Such a proof (for part (a)) is given in the author's recent paper [23], using an interpretation of $d_{\zeta}$ in terms of the homomorphism from $\mathcal{H}$ to Lusztig's "asymptotic algebra" $J$.
} 
4.2. For any $n \geq 1$ we define an element $c_{n} \in \mathcal{H}_{n}$ recursively as follows:

$$
c_{1}=T_{1}, \quad c_{n}=c_{n-1}+\zeta^{-2(n-1)} T_{s_{n-1}} \cdots T_{s_{2}} T_{s_{1}}^{2} T_{s_{2}} \cdots T_{s_{n-1}} \quad(n \geq 2) .
$$

It is not difficult to check that $c_{n}$ lies in the center of $\mathcal{H}_{n}$. This allows us to define linear operators $i$-res $n: R_{0}\left(\mathbb{C H}_{n}\right) \rightarrow R_{0}\left(\mathbb{C H}_{n-1}\right)(0 \leq i \leq d-1)$ as follows. Let $V$ be a simple $\mathbb{C H}_{n}$-module and $c \in \mathbb{C}$ be the scalar by which $c_{n}$ acts on $V$. We set

$$
V^{(i)}=\left\{v \in V \mid\left(c_{n-1}-c+\zeta^{2 i}\right)^{m} v=0 \text { for some } m \geq 1\right\} .
$$

Since $c_{n-1}$ lies in the center of $\mathcal{H}_{n-1}$, the space $V^{(i)}$ is a $\mathbb{C H}_{n-1}$-module; moreover, we have $V=\bigoplus_{i} V^{(i)}$, see [2, Prop. 2.1]. Now $i-$ res $_{n}$ is defined by sending the class of $V$ to the class of $V^{(i)}{ }^{5}$ Using the description of $\mathcal{K}_{n}$ as dual space in (4.1) we obtain a map $i$-res ${ }_{n}^{\text {tr }}: \mathcal{K}_{n-1} \rightarrow \mathcal{K}_{n}$. Considering all algebras $\mathcal{H}_{n}$ at the same time, we obtain linear operators $i$-res ${ }^{\text {tr }}: \mathcal{K} \rightarrow \mathcal{K}(0 \leq i \leq d-1)$ such that $i$-res ${ }^{\text {tr }}\left(\mathcal{K}_{n-1}\right) \subseteq \mathcal{K}_{n}$ for all $n$, where

$$
\mathcal{K}:=\bigoplus_{n \geq 0} \mathcal{K}_{n} \subseteq \mathcal{F}:=\bigoplus_{n \geq 0} \mathcal{F}_{n}, \quad \text { the so-called "Fock space". }
$$

Now we bring into play one further object, the Kac-Moody algebra $\widehat{\mathfrak{s l}}_{d}$ of type $A_{d-1}^{(1)}$ and its universal enveloping algebra $U\left(\widehat{\mathfrak{s l}}_{d}\right)$; (see [37]). There exists an action of $U\left(\widehat{\mathfrak{s l}}_{d}\right)$ on $\mathcal{F}$ such that $\mathcal{K}$ is an invariant subspace on which $f_{i}$ acts as $i$-res ${ }^{\text {tr }}$, where $f_{i}$ is a Chevalley generator for the negative part $U^{-}\left(\widehat{\mathfrak{s l}}_{d}\right)$. This action lifts in fact to the corresponding quantized universal enveloping algebra. (This is due to Hayashi; see the description by Misra and Miwa [48].) The second statement in the following result is [39, Conjecture 6.9(ii)].

TheOREM 4.3 (Ariki [2]). - ${ }^{6}$ The subspace $\mathcal{K} \subseteq \mathcal{F}$ is an integrable highest weight module with highest weight vector $[\emptyset]$. Its basis $\left\{P_{\mu}\right\}$, defined in terms of the matrices $D_{n}^{\zeta}$, is the canonical basis of $\mathcal{K}$ as an $U\left(\widehat{\mathfrak{s l}}_{d}\right)$-module (in the sense of Kashiwara and Lusztig).

For an introduction to canonical bases, see [46]; note, however, that the results presented there are needed in the generality of Lusztig [42, 43]. Lascoux, Leclerc, Thibon [39] also describe an efficient algorithm for computing the canonical basis of $\mathcal{K}$, which is implemented in Mathas' GAP package SPECHT [52], and in Veigneau's package ACE [54].

Ariki [2] proves in fact a more general version of the above result, which is also valid for Iwahori-Hecke algebras of type $B_{n}$ and for the cyclotomic Hecke algebras associated with $(\mathbb{Z} / m \mathbb{Z}) \rtimes \mathfrak{S}_{n}$ (see [3]). We then obtain even new results about the classification of the simple modules for these algebras (see Mathas [45]).

\footnotetext{
${ }^{5}$ If $d=p$ is a prime then, on the level of the symmetric groups, these operators have an interpretation in terms of induction/restriction of modules and sorting into $p$-blocks (see [33, (6.3.17)]). This also works on the level of $\mathcal{H}_{n}$, where $\zeta$ plays the role of the prime $p$. This follows from [35, Theorem 4.29] (the "Nakayama rule" for $\zeta$-blocks of $\mathcal{H}_{n}$ ) and the general results on representations of $\mathcal{H}_{n}$ in [16].

${ }^{6}$ In October 1995, Grojnowski also announced a proof of this result and of Theorem 4.7 below (following the ideas in his note [28]), as well as an extension to Jantzen filtrations, as suggested in [39, §9]. At the time of this writing, written proofs have not yet appeared.
} 
The idea of Ariki's proof is to establish a link between $U^{-}\left(\widehat{\mathfrak{s l}}_{d}\right)$ itself and a class of simple modules for an affine Hecke algebra (of which $\mathcal{H}_{n}$ is a quotient). The advantage of working with affine Hecke algebras is that the simple modules can be described geometrically via the "Deligne-Langlands conjecture"; see [38] and [11]. Our intention here is not to give a survey on representations of affine Hecke algebras; we shall use [11, Chap. 7 and 8$]$ as a reference for the facts about $\mathbf{H}_{n}$ that we shall need.

4.4. Let $\left\{\varepsilon_{1}, \ldots, \varepsilon_{n}\right\}$ be the standard basis of $\mathbb{C}^{n}$ and $X=\mathbb{Z} \varepsilon_{1}+\cdots+\mathbb{Z} \varepsilon_{n}$. Then $\left\{\alpha_{i}=\varepsilon_{i}-\varepsilon_{i+1}\right\}_{1 \leq i \leq n-1}$ is the set of simple roots in a root system of type $A_{n-1}$. Its Weyl group is the symmetric group $\mathfrak{S}_{n}$, acting by permutation on the basis vectors $\varepsilon_{i}$. The semidirect product $\mathbf{W}:=X \rtimes \mathfrak{S}_{n}$ is the extended affine Weyl group of type $A_{n-1}^{(1)}$.

The corresponding affine Hecke algebra is a free $A$-module $\mathbf{H}_{n}$ with a basis $\left\{\mathbf{T}_{x, w} \mid x \in\right.$ $\left.X, w \in \mathfrak{S}_{n}\right\}$ and the following rules for the multiplication: the map $T_{w} \mapsto \mathbf{T}_{0, w}$ is an algebra homomorphism, we have $\mathbf{T}_{x+y, w}=\mathbf{T}_{x, 1} \mathbf{T}_{y, w}$ for all $x, y \in X$ and $w \in \mathfrak{S}_{n}$, and we have the following Bernstein relations (see [11, Def. 7.1.9 and Lemma 7.1.10]):

$$
\mathbf{T}_{0, s_{\imath}} \mathbf{T}_{s_{i}(x), 1}-\mathbf{T}_{x, 1} \mathbf{T}_{0, s_{i}}=\left(1-v^{2}\right) \frac{\mathbf{T}_{x, 1}-\mathbf{T}_{s_{i}(x), 1}}{1-\mathbf{T}_{-\alpha_{i}, 1}}
$$

(Since $s_{i}$ acts as a reflection on $X$, the right hand side is a well-defined element of $\mathbf{H}_{n}$.)

LEMma 4.5. - We have a surjective $A$-algebra homomorphism $\pi_{n}: \mathbf{H}_{n} \rightarrow \mathcal{H}_{n}$, with

$$
\mathbf{T}_{0, w} \mapsto T_{w} \quad\left(w \in \mathfrak{S}_{n}\right), \quad \mathbf{T}_{\varepsilon_{i}, 1} \mapsto v^{2(i-1)}\left(T_{s_{1}} \cdots T_{s_{i-1}}\right)^{-1}\left(T_{s_{i-1}} \cdots T_{s_{1}}\right)^{-1} \quad(1 \leq i \leq n) .
$$

First note that, since $v$ is invertible in $A$, the elements $T_{s_{i}}$ are units in $\mathcal{H}_{n}$; we have $T_{s_{i}}^{-1}=v^{-2} T_{s_{i}}+\left(v^{-2}-1\right) T_{1}$ for $1 \leq i \leq n-1$. The fact that the above map defines a homomorphism is proved by checking the above defining relations.

4.6. As we did for $\mathcal{H}_{n}$, we write $\mathbb{C H}_{n}=\mathbb{C} \otimes_{A} \mathbf{H}_{n}$ where $\mathbb{C}$ is regarded as an $A$-module via the specialization $A \rightarrow \mathbb{C}, v \mapsto \zeta$. We shall be concerned exclusively with the algebra $\mathbb{C H}_{n}$ and its simple modules. The above construction shows that $\mathbb{C H}_{n}$ has countable dimension over $\mathbb{C}$. Moreover, $\mathbb{C H}_{n}$ is finitely generated over its center (see [11, Prop. 7.1.14]). These two facts imply that every simple $\mathbb{C H}_{n}$-module is finite-dimensional. Let $R_{0}\left(\mathbb{C H}_{n}\right)$ be the Grothendieck group of finite-dimensional $\mathbb{C H}_{n}$-modules.

We now single out a finitely generated subgroup of $R_{0}\left(\mathbb{C H}_{n}\right)$. Let $\lambda=\left(\lambda_{1}, \ldots, \lambda_{l}\right)$ be any composition of $n$ (i.e., we have $\lambda_{i}>0$ for all $i$, and $\sum_{i} \lambda_{i}=n$ ). Let $\mathbb{C H}_{n} \subseteq \mathbb{C H}_{n}$ be the subalgebra generated by $\mathbf{T}_{0, s_{2}}\left(\right.$ for $i \neq \lambda_{1}, \lambda_{1}+\lambda_{2}, \ldots$ ) and all $\mathbf{T}_{x, 1}$ (for $x \in X$ ). Assume we have a representation $\varphi: \mathbb{C}_{n} \rightarrow \mathbb{C}$ with $\varphi\left(\mathbf{T}_{0, s_{i}}\right)=\zeta^{2}$ for all $i$, and where $\varphi\left(\mathbf{T}_{x, 1}\right)$ is a power of $\zeta^{2}$ for all $x \in X$. Let $\mathbb{C}_{\varphi}$ be the corresponding $\mathbb{C H}_{n}$-module and set

$$
M_{\lambda, \varphi}:=\mathbb{C H}_{n} \otimes_{\mathbb{C}_{n}} \mathbb{C}_{\varphi} .
$$

Then we let $R_{0}^{\zeta}\left(\mathbb{C H}_{n}\right)$ be the subgroup of $R_{0}\left(\mathbb{C H}_{n}\right)$ which is generated by the composition factors of $M_{\lambda, \varphi}$, for all $\lambda, \varphi$ as above. - Note that $\pi_{n}$ induces an injective map 
$\dot{\pi}_{n}: R_{0}\left(\mathbb{C H}_{n}\right) \rightarrow R_{0}\left(\mathbb{C H}_{n}\right)$, whose image is contained in $R_{0}^{\zeta}\left(\mathbb{C H}_{n}\right)$. Moreover, there are also operators $i$-res ${ }_{n}: R_{0}\left(\mathbb{C H}_{n}\right) \rightarrow R_{0}\left(\mathbb{C H}_{n-1}\right)$ defined in a similar way as above, using the central element $\mathbf{T}_{-\varepsilon_{1}, 1}+\cdots+\mathbf{T}_{-\varepsilon_{n}, 1} \in \mathbf{H}_{n}$. Note that this element maps to $c_{n}$ under $\pi_{n}$, and we have the commutation rule: $\dot{\pi}_{n-1} \circ\left(i-\operatorname{res}_{n}\right)=\left(i-\operatorname{res}_{n}\right) \circ \dot{\pi}_{n}$.

We can now "lift" the desired statement in Theorem 4.3 to a statement about $U^{-}\left(\widehat{\mathfrak{s l}}_{d}\right)$ and $\bigoplus_{n \geq 0} R_{0}^{\zeta}\left(\mathbb{C H}_{n}\right)$. Note that the canonical basis of $\mathcal{K}$ is in fact defined as $\{b . \emptyset \mid b \in$ B $\} \backslash\{0\}$, where B is the canonical basis of $U^{-}\left(\widehat{\mathfrak{s l}}_{d}\right)$, see [42, Cor. 11.10]. Ariki shows that Theorem 4.3 is a consequence of the following result:

Theorem 4.7 (Ariki [2, Prop. 4.3]). - There exists a $\mathbb{C}$-linear isomorphism

$$
\psi: U^{-}\left(\widehat{\mathfrak{s l}}_{d}\right) \longrightarrow \bigoplus_{n \geq 0}\left(\mathbb{C} \otimes_{\mathbb{Z}} R_{0}^{\varsigma}\left(\mathbb{C H}_{n}\right)\right)^{*}
$$

which commutes with multiplication by any $f_{i}$ on the left hand side and the action of $i$ res $^{\text {tr }}$ on the right hand side. Furthermore, $\psi(\mathbf{B})$ is the set obtained by taking in the $n$-th summand the dual basis of the standard basis given by the simple modules.

\section{AFFINE HECKE ALGEBRAS AND CANONICAL BASES}

The objective of this section is to present the main ideas behind Ariki's proof of Theorem 4.7. (We do not claim to have verified all the details.) It is based on:

(1) A classification of the simple $\mathbb{C H}_{n}$-modules in terms of a formula describing their multiplicities in certain "standard" modules; see [11] and Theorem 5.2 below.

(2) Lusztig's geometric description of the canonical basis of $U^{-}\left(\widehat{\mathfrak{s l}}_{d}\right)$; see $[42,43]$ and (5.4) below.

(3) A version of Theorem 4.7 for the case where $\zeta$ has infinite order and a specialization argument; see (5.6) and (5.8) below.

Throughout this section, $\zeta$ may be any non-zero complex number. We consider the affine Hecke algebra $\mathbb{C H}_{n}$ corresponding to the specialization $A \rightarrow \mathbb{C}, v \mapsto \zeta$.

The simple modules of $\mathbb{C H}_{n}$, where $\zeta$ is not a root of unity, have been classified in a geometric framework by Kazhdan and Lusztig [38]. We begin by discussing an extension of this result to the general case.

5.1. Let $G=\mathrm{GL}_{n}(\mathbb{C})$ and $T \subseteq G$ be the maximal torus consisting of diagonal matrices. Then the $\mathbb{Z}$-lattice $X$ in (4.4) can be interpreted as $\operatorname{Hom}\left(T, \mathbb{C}^{\times}\right)$, where $\varepsilon_{i}$ corresponds to the map which takes an element of $T$ to its $i$-th diagonal entry.

Let $s \in T$. Then the pair $(s, \zeta)$ determines an algebra homomorphism $\mathrm{Z}\left(\mathbf{H}_{n}\right) \rightarrow \mathbb{C}$ (where $\mathrm{Z}\left(\mathbf{H}_{n}\right)$ is the center of $\mathbf{H}_{n}$ ), which depends only on the $G$-conjugacy class of $s$. Regarding $\mathbb{C}$ as a $\mathrm{Z}\left(\mathbf{H}_{n}\right)$-module via this homomorphism, we form the tensor product

$$
\mathbf{H}_{n}^{(s, \zeta)}:=\mathbb{C} \otimes_{\mathbf{Z}\left(\mathbf{H}_{n}\right)} \mathbf{H}_{n}, \quad \text { the "specialization of } \mathbf{H}_{n} \text { at }(s, \zeta) \text { "; }
$$


see [11, Def. 8.1.1]. This is a finite-dimensional $\mathbb{C}$-algebra, and we have a natural algebra homomorphism $\mathbb{C H}_{n} \rightarrow \mathbf{H}_{n}^{(s, \zeta)}$. By [11, Cor. 8.1.4], the action of $\mathbb{C H}_{n}$ on any simple module factors through $\mathbf{H}_{n}^{(s, \zeta)}$, for some $s$. Thus, as far as simple modules are concerned, we are reduced to work with $\mathbf{H}_{n}^{(s, \zeta)}$.

Now consider the conjugation action of $G$ on the Lie algebra $\mathfrak{g l}_{n}(\mathbb{C})$. We set

$$
\mathcal{N}_{\zeta}^{s}:=\left\{x \in \mathfrak{g l}_{n}(\mathbb{C}) \mid x \text { nilpotent and } s x s^{-1}=\zeta^{2} x\right\} .
$$

Then $C_{G}(s)$ acts on $\mathcal{N}_{\zeta}^{s}$ with only finitely many orbits (see [11, Prop. 8.1.17]). Now take $x \in \mathcal{N}_{\zeta}^{s}$ and consider $\mathcal{B}_{x}^{s}$, the variety of all flags which are fixed by $s$ and by $x$. By [11, Remark 8.1.7], this variety is non-empty. Then one can construct a natural $\mathbf{H}_{n}^{(s, \zeta)}$-action on $H_{*}\left(\mathcal{B}_{x}^{s}\right)$, the Borel-Moore homology of $\mathcal{B}_{x}^{s}$ with coefficients in $\mathbb{C}$. This is done through a $K$-theoretic interpretation of the algebra $\mathbf{H}_{n}^{(s, \zeta)}$, which is explained in [11, Prop. 8.15].

The following result has been called by Zelevinski the " $p$-adic analogue of the KazhdanLusztig formula for multiplicities of Verma modules" (see [11, p.17]). Its proof, to be found in [11, Theorem 8.6.23], uses among other things the "Decomposition Theorem" of Bernstein, Beilinson, Deligne [4] and, hence, ultimately rests on the Weil conjectures.

THEOREM 5.2. - The simple $\mathbf{H}_{n}^{(s, \zeta)}$-modules occuring in $H_{*}\left(\mathcal{B}_{x}^{s}\right)$ have a natural labelling by $C_{G}(s)$-orbits in $\mathcal{N}_{\zeta}^{s}$ (but not all orbits need occur). Let $O$ be such an orbit and $L_{O}^{s}$ the corresponding simple module. Then the multiplicity of $L_{O}^{s}$ in $H_{*}\left(\mathcal{B}_{x}^{s}\right)$ is given by $\sum_{i>0} \operatorname{dim} H^{i}\left(\iota_{x}^{!} \mathrm{IC}(O, \mathbb{C})\right)$, where $\iota_{x}:\{x\} \hookrightarrow \mathcal{N}_{\zeta}^{s}$ and $\operatorname{IC}(O, \mathbb{C})$ denotes the DeligneGoreski-MacPherson intersection cohomology complex on the closure of $O$.

Thus, we have a way to get hold of the simple $\mathbb{C H}_{n}$-modules by decomposing the "standard" modules $H_{*}\left(\mathcal{B}_{x}^{s}\right)$ into simple modules, where the multiplicities are, in principle, computable in terms of the geometry of $G$. Note, however, that the problem of characterizing the "missing" orbits remains to be solved. If $\zeta$ is not a root of unity, a solution is given in [38] and the "non-vanishing result" in [11, §8.8]: in this case, no orbit is "missing". (Grojnowski [28] announces a solution for this problem in general; see also Vigneras $[56, \S 2]$.) We will come back to this point in (5.6) and Proposition 5.9 below.

Now the "standard" modules $H_{*}\left(\mathcal{B}_{x}^{s}\right)$ can be identified with induced modules as in (4.6). To see this, we replace $s, x$ by suitable $G$-conjugates so that $x$ is in Jordan normal form (and we still have $s \in T$ ). Let $\lambda$ be the composition of $n$ given by the sizes of the Jordan blocks, and $\mathbb{C}_{n} \subseteq \mathbb{C H}_{n}$ the corresponding subalgebra as in (4.6). We have a representation $\varphi_{s}: \mathbb{C H}_{n} \rightarrow \mathbb{C}$ with $\varphi_{s}\left(\mathbf{T}_{0, s_{i}}\right)=\zeta^{2}$ and $\varphi_{s}\left(\mathbf{T}_{y, 1}\right)=y(s)$ for all $y \in X$. Using the induction theorem $[38, \S 6]$, Ariki obtains the following result in [2, Theorem 3.2]:

THEOREM 5.3. - With the above notation, we have an equality $\left[H_{*}\left(\mathcal{B}_{x}^{s}\right)\right]=\left[M_{\lambda, \varphi_{s}}\right]$ in the Grothendieck group of $\mathbb{C H}_{n}$-modules, where the module structure on the left hand side is induced from that on $\mathbf{H}_{n}^{(s, \zeta)}$ defined in (5.1). 
We now turn to the second ingredient, the theory of canonical bases for (quantized) universal enveloping algebras (see [46]). We shall work exclusively with Lusztig's geometric description $[42,43]$. Depending on whether $\zeta$ has finite or infinite order, we consider an affine algebra of finite or infinite rank. We begin with the case of finite rank.

5.4. The canonical basis $\mathbf{B}$ of $U^{-}\left(\widehat{\mathfrak{s l}}_{d}\right)$ is defined in terms of a class of perverse sheaves on certain varieties attached to the affine Dynkin diagram of type $A_{d-1}^{(1)}$. The details of this construction which are important for us can be summarized as follows (see [2, (4.3)]).

There is a natural grading $U^{-}\left(\widehat{\mathfrak{s l}}_{d}\right)=\bigoplus_{\nu} U_{\nu}^{-}$where $\nu$ are functions on the vertices $\{0, \ldots, d-1\}$ of the diagram of type $A_{d-1}^{(1)}$ with values in $\mathbb{N}_{0}$ and $U_{\nu}^{-}$are finite-dimensional. The canonical basis $\mathbf{B}$ is compatible with this grading, i.e., we have $\mathbf{B}=\coprod_{\nu} \mathbf{B}_{\nu}$ where $\mathbf{B}_{\nu}=\mathbf{B} \cap U_{\nu}^{-}$. Now assume that $\zeta$ has order $2 d$. Then any $\nu$ as above determines a semisimple element $s \in G=\mathrm{GL}_{n}(\mathbb{C})$ (well-defined up to conjugacy), where $n=\sum_{i=0}^{d-1} \nu(i)$ : take the diagonal matrix whose eigenvalues are powers of $\zeta^{2}$ and the dimension of the $\zeta^{2 i}$ eigenspace is given by $\nu(i)$. Then, by [42, (12.14) and §15] and [43], the elements of $\mathbf{B}_{\nu}$ are naturally in bijection to a set of $C_{G}(s)$-orbits on $\mathcal{N}_{\zeta}^{s}$ (but not all orbits need occur). Thus, we are in a situation similar to that in Theorem 5.2, but now Lusztig has given an explicit description of those orbits which do correspond to elements in $\mathbf{B}_{\nu}$ : these are the so-called "aperiodic" orbits, see the definition in $[42, \S 15]$.

Let $O \subseteq \mathcal{N}_{\zeta}^{s}$ be an "aperiodic" orbit and denote by $b_{O}^{\nu}$ the corresponding canonical basis element. This element is defined in terms of the Deligne-Goreski-MacPherson intersection cohomology complex on the closure of $O$. Taking the alternating sum of the dimensions of the stalks of the cohomology sheaves of such a complex at points in $\mathcal{N}_{\zeta}^{s}$, we can regard $b_{O}^{\nu}$ as a $\mathbb{C}$-valued function on $\mathcal{N}_{\zeta}^{s}$ which is constant on the orbits of $C_{G}(s)$. Furthermore, for any orbit $O^{\prime} \subseteq \mathcal{N}_{\zeta}^{s}$, we denote by $p_{O^{\prime}}^{\nu}$ the function which takes the value 1 on $O^{\prime}$ and 0 otherwise. Then we have equations

$$
b_{O}^{\nu}=\sum_{O^{\prime}} c_{O O^{\prime}}^{s} p_{O^{\prime}}^{\nu}, \quad \text { where } \quad c_{O O^{\prime}}^{s}:=\sum_{i \geq 0} \operatorname{dim} H^{i}\left(\iota_{x}^{!} \mathrm{IC}(O, \mathbb{C})\right) \quad \text { for } x \in O^{\prime} .
$$

The set $\left\{p_{O}^{\nu}\right\}$ will be called a "PBW-type basis" of $U^{-}\left(\widehat{\mathfrak{s l}}_{d}\right)$.

REMARK 5.5. - Ariki notes in $[2,(4.3)]$ that the results in (5.4) actually remain valid when we set $d=\infty$. In this case, we are working with the infinite rank affine algebra $\mathfrak{s l}_{\infty}$, of type $A_{\infty}$ (see $\left.[37, \S 7.11]\right)$. The canonical basis $\mathbf{B}_{\infty}$ of $U^{-}\left(\mathfrak{s l}_{\infty}\right)$ can be described as the union of the canonical bases over all finite rank algebras of type $A_{r}(r \geq 1)$. Again, we have a partition $\mathbf{B}_{\infty}=\coprod_{\nu} \mathbf{B}_{\infty, \nu}$ where $\nu$ are functions on the vertices of the diagram of type $A_{\infty}$ with only finitely many non-zero values in $\mathbb{N}$. By the same procedure as in (5.4), but where $\zeta$ has infinite order, any such $\nu$ determines an $n$ and a semisimple element $s \in G=\mathrm{GL}_{n}(\mathbb{C})$. Now we have a natural bijection between $\mathbf{B}_{\infty, \nu}$ and the set of all $C_{G}(s)$-orbits in $\mathcal{N}_{\zeta}^{s}$; see [42, (12.14) and §14]. Furthermore, we have relations as in (5.4) expressing the elements of $\mathbf{B}_{\infty}$ in terms of the elements of a "PBW-type basis" $\left\{p_{O}^{s}\right\}$. 
We have now geometric descriptions of the simple modules for affine Hecke algebras on the one hand and of canonical bases on the other hand. The next step consists of bringing these two descriptions together. We first do this in the case where $\zeta$ has infinite order.

5.6. A version of Theorem 4.7 for the case where $\zeta$ has infinite order. Assume that $\zeta$ has infinite order. Then, in the set-up of Theorem 5.2, all $C_{G}(s)$-orbits $O \subseteq \mathcal{N}_{\zeta}^{s}$ give rise to non-zero simple modules $L_{O}^{s}$; see [38] and the "non-vanishing result" in [11, $\S 8.8]$. Hence, in this case, we have a natural bijection between the simple $\mathbb{C H}_{n}$-modules (up to isomorphism) and the set of all pairs $(s, O)$ where $s \in G$ is semisimple (up to conjugation) and $O$ is a $C_{G}(s)$-orbit in $\mathcal{N}_{\zeta}^{s}$. (This is the "Deligne-Langlands conjecture" for affine Hecke algebras of type $A_{n-1}^{(1)}$.)

Next note that, setting formally $d=\infty$, the definitions in (4.6) remain valid (it is not required there that $\zeta$ has finite order). Then we observe that the subgroup $R_{0}^{\zeta}\left(\mathbb{C H}_{n}\right) \subseteq$ $R_{0}\left(\mathbb{C H}_{n}\right)$ is generated by the classes of simple modules arising from those algebras $\mathbf{H}_{n}^{(s, \zeta)}$ where all eigenvalues of $s$ are powers of $\zeta^{2}$. (See [2, (4.1), (4.2)]; note that the transition between the settings in (4.6) and (5.1) is provided by Theorem 5.3.) Thus, we can define a $\mathbb{C}$-linear isomorphism

$$
\psi_{\infty}: U^{-}\left(\mathfrak{s l}_{\infty}\right) \stackrel{\sim}{\longrightarrow} \bigoplus_{n \geq 0}\left(\mathbb{C} \otimes_{\mathbb{Z}} R_{0}^{\zeta}\left(\mathbb{C H}_{n}\right)\right)^{*}
$$

as follows. Let $\mathbf{B}_{\infty}=\coprod_{\nu} \mathbf{B}_{\infty, \nu}$ be the canonical basis of $U^{-}\left(\mathfrak{s l}_{\infty}\right)$ as in Remark 5.5. Let $b \in \mathbf{B}_{\infty, \nu}$. Then $b=b_{O}^{\nu}$ for a unique orbit $O \subseteq \mathcal{N}_{\zeta}^{s}$, where $n$ and $s \in \mathrm{GL}_{n}(\mathbb{C})$ are determined by $\nu$. The orbit $O$ gives rise to a simple $\mathbb{C H}_{n}$-module $L_{O}^{s}$ as in Theorem 5.2, and we set $\psi_{\infty}(b)=\left[L_{O}^{s}\right]^{*}$, the dual element in $\left(\mathbb{C} \otimes_{\mathbb{Z}} R_{0}^{\zeta}\left(\mathbb{C H}_{n}\right)\right)^{*}$. Note that, since the spaces $R_{0}^{\zeta}\left(\mathbb{C H}_{n}\right)$ have infinite rank, the map $\psi_{\infty}$ only becomes an isomorphism when we actually work in suitable completions of the above vector spaces, where formal infinite sums of the basis elements are allowed. Keeping this in mind, we can now state:

Proposition 5.7. - Recall that $\zeta$ is assumed to have infinite order. Then the isomorphism $\psi_{\infty}$ of (5.6) commutes with multiplication by any $f_{i}$ (a Chevalley generator of $\left.U^{-}\left(\mathfrak{s l}_{\infty}\right)\right)$ on the left hand side and the action of $i$-res ${ }^{\text {tr }}$ on the right hand side.

For the proof, we fix $\nu$ and a corresponding semisimple element $s \in \mathrm{GL}_{n}(\mathbb{C})$. Then we have equations $b_{O}^{\nu}=\sum_{O^{\prime}} c_{O O^{\prime}}^{s} p_{O^{\prime}}^{s}$ where $O, O^{\prime}$ are orbits in $\mathcal{N}_{\zeta}^{s}$. By Theorem 5.2 we also have equations $\left[M_{O}^{s}\right]=\sum_{O^{\prime}} c_{O^{\prime} O}^{s}\left[L_{O^{\prime}}^{s}\right]$, where $O, O^{\prime}$ are orbits in $\mathcal{N}_{\zeta}^{s}$ and where we set $M_{O}^{s}=H_{*}\left(\mathcal{B}_{x}^{s}\right)$ for $x \in O$. Now, if we choose an ordering of the orbits $O$ which is compatible with the closure relation, we see that the matrix $\left(c_{O O^{\prime}}^{s}\right)$ is square and triangular with 1 along the diagonal. It follows that the classes of the modules $M_{O}^{s}$ also form a basis of $\mathbb{C} \otimes_{\mathbb{Z}} R_{0}^{\zeta}\left(\mathbb{C H}_{n}\right)$. Passing to the dual spaces yields that

$$
\sum_{O^{\prime}} c_{O O^{\prime}}^{s} \psi_{\infty}\left(p_{O^{\prime}}^{\nu}\right)=\psi_{\infty}\left(b_{O}^{\nu}\right)=\left[L_{O}^{s}\right]^{*}=\sum_{O^{\prime}} c_{O O^{\prime}}^{s}\left[M_{O^{\prime}}^{s}\right]^{*}
$$


where $\left\{\left[M_{O}^{s}\right]^{*}\right\}$ are the elements of $\mathbb{C} \otimes_{\mathbb{Z}} R_{0}^{\zeta}\left(\mathbb{C H}_{n}\right)$ which are dual to the "standard" modules. Hence, we deduce that $\psi_{\infty}\left(p_{O}^{\nu}\right)=\left[M_{O}^{s}\right]^{*}$ for all $O$, and we see that it is sufficient to prove the desired compatibility properties on the level of the "PBW-type basis" on the one hand and the "standard" modules on the other hand.

The multiplication of a "PBW-type basis" element by a generator $f_{i}$ can be described explicitly in purely combinatorial terms (see [2, Lemma 4.2]). We compare this with the action of $i$-res ${ }^{\text {tr }}$, by describing explicitly the $i$-restriction of a "standard" module for $\mathbb{C H}_{n}$ as a sum of "standard" modules for $\mathbb{C H}_{n-1}$. In order to achieve this, Ariki uses the following argument: we know by Theorem 5.3 that a "standard" module for $\mathbf{C H}_{n}$ is given in terms of a certain induced module. This interpretation allows us to regard that "standard" module as a module for a factor algebra of $\mathbb{C H}_{n}$, namely a cyclotomic algebra associated with the group $(\mathbb{Z} / m \mathbb{Z}) \rtimes \mathfrak{S}_{n}$ for a suitable $m$ and a suitable choice of parameters (see the proof of [2, Prop. 4.3(1)]). Now, for these algebras, the restriction of modules is determined combinatorially in terms of a "branching rule", see [2, Prop. 2.1]. This yields the desired compatibility and completes the proof of Proposition 5.7.

5.8. Passage to the case where $\zeta$ is a primitive root of unity of order $2 d$. Assume that $\zeta$ has order $2 d$. In order to have separate notations, we also let $\zeta^{\prime}$ be a nonzero complex number of infinite order and denote the corresponding affine Hecke algebra by $\mathbb{C H}_{n}^{\prime}$. Consider the isomorphism of (5.6)

$$
\psi_{\infty}: U^{-}\left(\mathfrak{s l}_{\infty}\right) \stackrel{\sim}{\longrightarrow} \bigoplus_{n \geq 0}\left(\mathbb{C} \otimes_{\mathbb{Z}} R_{0}^{\zeta^{\prime}}\left(\mathbb{C H}_{n}^{\prime}\right)\right)^{*} .
$$

By [13], we can realize $U^{-}\left(\widehat{\mathfrak{s l}}_{d}\right)$ inside $U^{-}\left(\mathfrak{s l}_{\infty}\right)$ by a "folding" procedure. On the other hand, by a specialization argument, $R_{0}^{\zeta}\left(\mathbb{C H}_{n}\right)$ can be regarded as a quotient of $R_{0}^{\zeta^{\prime}}\left(\mathbb{C H}_{n}^{\prime}\right)$ and, hence, $\left(\mathbb{C} \otimes_{\mathbb{Z}} R_{0}^{\zeta}\left(\mathbb{C H}_{n}\right)\right)^{*}$ can be identified with a subspace of $\left(\mathbb{C} \otimes_{\mathbb{Z}} R_{0}^{\zeta^{\prime}}\left(\mathbb{C H}_{n}^{\prime}\right)\right)^{*}$. Then Ariki shows in [2, p. 804] that, under these identifications, the image of $U^{-}\left(\widehat{\mathfrak{s l}}_{d}\right)$ under $\psi_{\infty}$ is contained in $\bigoplus_{n \geq 0}\left(\mathbb{C} \otimes_{\mathbb{Z}} R_{0}^{\zeta}\left(\mathbb{C H}_{n}\right)\right)^{*}$. Hence, by restriction, $\psi_{\infty}$ gives rise to an injective map

$$
\psi_{d}: U^{-}\left(\widehat{\mathfrak{s l}}_{d}\right) \hookrightarrow \bigoplus_{n \geq 0}\left(\mathbb{C} \otimes_{\mathbb{Z}} R_{0}^{\zeta}\left(\mathbb{C H}_{n}\right)\right)^{*} .
$$

The effects of specialization and "folding" on the standard modules and on the "PBWtype basis", respectively, can be described explicitly, and this can be used to show that $\psi_{d}$ commutes with multiplication by any $f_{i}$ (a Chevalley generator of $U^{-}\left(\widehat{\mathfrak{s l}}_{d}\right)$ ) on the left hand side and the action of $i-$ res $^{\text {tr }}$ on the right hand side. By a similar argument as in the proof of Proposition 5.7, one then also shows that the elements of the canonical basis of $U^{-}\left(\widehat{\mathfrak{s l}}_{d}\right)$ are mapped to the duals of simple modules as required in Theorem 4.7 (see [2, Prop. 4.3(2)]). Hence, to complete the proof of Theorem 4.7, it is sufficient to show that $\psi_{d}$ is surjective. For this purpose, we need a characterization of the "missing" orbits in Theorem 5.2. This is provided by the following result. 
Proposition 5.9. - Assume that $\zeta$ is a primitive $2 d$-th root of unity. Then, in the setup of Theorem 5.2, the $C_{G}(s)$-orbit $O \subseteq \mathcal{N}_{\zeta}^{s}$ gives rise to a non-zero simple $\mathbf{H}_{n}^{(s, \zeta)}$-module $L_{O}^{s}$ if and only if $O$ is "aperiodic" (in the sense of (5.4)).

Ariki observes that a proof of this result can be achieved as follows. First we note that the construction of $L_{O}^{s}$, as explained in [11, p. 444 and Theorem 8.6.12], is just a special case of the geometric construction of canonical basis elements in [42, §2]. In both cases, one has to consider certain varieties which map properly to $\mathcal{N}_{\zeta}^{s}$ and one has to decompose the direct image of the constant sheaf on such a variety into irreducible perverse sheaves. Now the known results on the canonical basis, see (5.4), imply that an orbit $O$ must be "aperiodic" if it gives rise to a non-zero simple module $L_{O}^{s}$. It remains to show that the rank of $R_{0}^{\zeta}\left(\mathbb{C H}_{n}\right)$ is equal to or bigger than the dimension of $U_{\nu}^{-}$. But this follows from the injectivity of the map $\psi_{d}$ in (5.8).

Thus, Proposition 5.9 and, hence, Theorem 4.7 are proved.

Acknowledgements. I am indebted to S. Ariki for patiently answering all my questions about his article [2]. Many thanks are due to I. Grojnowski for a number of helpful comments on his note [28]. I also wish to thank G. Hiss, B. Leclerc, J. Müller, and R. Rouquier for some useful discussions and critical comments.

\section{REFERENCES}

[1] D. ALVIS and G. LUSZTIG - The representations and generic degrees of the Hecke algebra of type $H_{4}$, J. reine angew. Math. 336 (1982), 201-212; correction: ibid. 449 (1994), 217-218.

[2] S. ARIKI - On the decomposition numbers of the Hecke algebra of $G(m, 1, n)$, J. Math. Kyoto Univ. 36 (1996), 789-808.

[3] S. ARIKI and K. KOIKE - A Hecke algebra of $(\mathbb{Z} / r \mathbb{Z})$ wreath $\mathfrak{S}_{n}$ and construction of its irreducible representations, Advances in Math. 106 (1994), 216-243.

[4] A.A. BEILInson, J. BERnSTEIN, P. DELIGNE - Faisceaux pervers, Astérisque 100 (1982).

[5] C.T. BENSON and C.W. CURTIS - On the degrees and rationality of certain characters of finite Chevalley groups, Trans. Amer. Math. Soc. 165 (1972), 251-273; corrections and additions, ibid. 202 (1975), 405-406.

[6] N. BOURBAKI - Groupes et algèbres de Lie, Chap. 4, 5 et 6, Hermann, Paris (1968).

[7] K. BREMKE - The decomposition numbers of Hecke algebras of type $F_{4}$ with unequal parameters, Manuscripta Math. 83 (1994), 331-346.

[8] M. BROUÉ and G. MALLE - Zyklotomische Heckealgebren, in: Représentations unipotentes génériques et blocs des groupes réductifs finis, Astérisque 212 (1993), 119-189. 
[9] R.W. CARTER - Finite groups of Lie type: Conjugacy classes and complex characters, Wiley, New York (1985).

[10] B.W. CHAR et. al. - Maple V, Language Reference Manual, Springer (1991); see also http://daisy.uwaterloo.ca

[11] N. CHRISS and V. GINZBURG - Representation theory and complex geometry, Birkhäuser, Boston (1997).

[12] C.W. CURTIS and I. REINER - Methods of Representation Theory Vol. I and II, Wiley, New York (1981 and 1987).

[13] E. DATE, M. JIMBO, M. KASHIWARA, T. MIWA - Transformation groups for soliton equations. Euclidean Lie algebras and reduction of KP hierarchy, Publ. RIMS 18 (1982), 1077-1110.

[14] R. DIPPER - Polynomial representations of finite general linear groups in nondescribing characteristic, in: G.O. Michler, C.M. Ringel (ed.), Representation theory of finite groups and finite-dimensional algebras, Progress in Math. 95 (1991), 344370, Birkhäuser, Boston.

[15] R. DIPPER, M. GECK, G. HISS, and G. MALLE - Representations of Hecke algebras and finite groups of Lie type, to appear in a proceedings volume on the DFG project "Algorithmic algebra and number theory".

[16] R. DIPPER and G.D. JAMES - Representations of Hecke algebras of the general linear groups, Proc. London Math. Soc. 52 (1986), 20-52.

[17] R. DIPPER and G.D. JAMES - The q-Schur algebra, Proc. London Math. Soc. 59 (1989), 23-50.

[18] R. DIPPER and G.D. JAMES - $q$-Tensor space and $q$-Weyl modules, Trans. Amer. Math. Soc. 327 (1991), 251-282.

[19] R. DIPPER, G.D. JAMES, and G.E. MURPHY - Hecke algebras of type $B_{n}$ at roots of unity, Proc. London Math. Soc. 70 (1995), 505-528.

[20] P. FLEISCHMANN - Projective simple modules of symmetric algebras and their specializations with applications to Hecke algebras, Arch. Math. 55 (1990), 247-258.

[21] M. GECK - Brauer trees of Hecke algebras, Comm. Algebra 20 (1992), 2937-2973.

[22] M. GECK - The decomposition numbers of the Hecke algebra of type $E_{6}$, Math. Comp. 61 (1993), 889-899.

[23] M. GECK - Kazhdan-Lusztig cells and decomposition numbers, Represent. Theory 2 (1998), 264-277 (electronic).

[24] M. GECK and G. HISS - Modular representations of finite groups of Lie type in non-defining characteristic, in: M. Cabanes (ed.), Finite reductive groups, related structures and representations, Progress in Math. 141 (1997), 195-249, Birkhäuser, Boston.

[25] M. GECK and K. LUX - The decomposition numbers of the Hecke algebra of type $F_{4}$, Manuscripta Math. 70 (1991), 285-306. 
[26] M. GECK and G. PFEIFFER - On the irreducible characters of Hecke algebras, Advances in Math. 102 (1993), 79-94.

[27] M. GECK and R. ROUQUIER - Centers and simple modules for Iwahori-Hecke algebras, in: M. Cabanes (ed.), Finite reductive groups, related structures and representations, Progress in Math. 141 (1997), 251-272, Birkhäuser, Boston.

[28] I. GROJNOWSKI - Representations of affine Hecke algebras (and quantum $G L_{n}$ ) at roots of unity, Internat. Math. Research Notices 5 (1994), 215-217.

[29] J. GRUBER and G. HISS - Decomposition numbers of finite classical groups for linear primes, J. reine angew. Math. 485 (1997), 55-91.

[30] A. GYOJA - Modular representation theory over a ring of higher dimension with applications to Hecke algebras, J. Algebra 174 (1995), 553-572.

[31] A. GYOJA - Cells and modular representations of Hecke algebras, Osaka J. Math. 33 (1996), 307-341.

[32] G.D. JAMES - The decomposition matrices of $G L_{n}(q)$ for $n \leq 10$, Proc. London Math. Soc. 60 (1990), 225-265.

[33] G.D. JAMES and A. KERBER - The representation theory of the symmetric group, Encyclopedia Math. 16, Addison-Wesley (1981).

[34] G.D. JAMES and A. MATHAS - Hecke algebras of type A with $q=-1$, J. Algebra 184 (1996), 102-158.

[35] G.D. JAMES and A. MATHAS - A q-analogue of the Jantzen-Schaper theorem, Proc. London Math. Soc. 74 (1997), 241-274.

[36] V.F.R. JONES - Hecke algebra representations of braid groups and link polynomials, Annals Math. 126 (1987), 335-388.

[37] V.G. KAC - Infinite dimensional Lie algebras, Cambridge University Press (1985).

[38] D. KAZHDAN and G. LUSZTIG - Proof of the Deligne-Langlands conjecture for Hecke algebras, Invent. Math. 87 (1987), 153-215.

[39] A. LASCOUX, B. LECLERC and J.Y. THIBON - Hecke algebras at roots of unity and crystal bases of quantum affine algebras, Comm. Math. Physics 181 (1996), 205-263; see also the announcement in C. R. Acad. Sci. Paris 321 (1995), 511-516.

[40] B. LECLERC and J.Y. THIBON - Canonical bases of $q$-deformed Fock spaces, Internat. Math. Research Notices 9 (1996), 447-456.

[41] G. LUSZTIG - On a theorem of Benson and Curtis, J. Algebra 71 (1981), 490-498.

[42] G. LUSZTIG - Quivers, perverse sheaves and quantized enveloping algebras, J. Amer. Math. Soc. 4 (1991), 365-421.

[43] G. LUSZTIG - Affine quivers and canonical bases, Publ. Math. IHES 76 (1992), 111-163.

[44] G. LUSZTIG - Classification of unipotent representations of simple p-adic groups, Internat. Math. Research Notices 11 (1995), 517-589. 
[45] A. MATHAS - Canonical bases and the decomposition matrices of Ariki-Koike algebras, preprint (1996).

[46] O. MATHIEU - Bases des représentations des groupes simples complexes (d'après Kashiwara, Lusztig, Ringel et al.), Sém. Bourbaki, 1990-91, exposé $\mathrm{n}^{\circ} 743$, Astérisque 201-202-203 (1991), 421-442.

[47] H. MATSUMURA - Commutative ring theory, Cambridge Studies in Advanced Math. 8, Cambridge Univerisity Press (1986).

[48] K.C. MISRA and T. MIWA - Crystal base of the basic representation of $U_{q}\left(\widehat{\mathfrak{s l}}_{n}\right)$, Comm. Math. Physics 134 (1990), 79-88.

[49] J. MÜLLER - Zerlegungszahlen für generische Iwahori-Hecke-Algebren von exzeptionellem Typ, Dissertation, RWTH Aachen (1995).

[50] J. MÜLLER - Decomposition numbers for generic Iwahori-Hecke algebras of non crystallographic type, J. Algebra 189 (1997), 125-149.

[51] C. PALLIKAROS - Representations of Hecke algebras of type $D_{n}$, J. Algebra 169 (1994), 20-48.

[52] M. SCHÖNERT et al. - GAP - Groups, algorithms, and programming, Lehrstuhl D für Mathematik, RWTH Aachen (1994), http://www.math.rwth-aachen.de/LDFM

[53] W. SOERGEL - Conjectures de Lusztig, Sém. Bourbaki, 1994-95, exposé nº 793, Astérisque 237 (1996), 75-85.

[54] S. VEIGNEAU - ACE - An algebraic combinatorics environment for the computer algebra system MAPLE, User's reference manual, Version 2.0, Institut Gaspard Monge (1996), http://weyl.univ-mlv.fr/ sv/ACE.

[55] M. VARAGNOLO and E. VASSEROT - Canonical bases and the Lusztig conjecture for quantized $S L(n)$ at roots of unity, preprint (1998).

[56] M.F. VIGNERAS - A propos d'une conjecture de Langlands modulaire, in: M. Cabanes (ed.), Finite reductive groups, related structures and representations, Progress in Math. 141 (1997), 415 - 452, Birkhäuser, Boston.

\author{
Meinolf GECK \\ UFR de Mathématiques \\ et UMR 7586 du CNRS \\ Université Paris 7 \\ 2 Place Jussieu \\ F-75251 Paris Cedex 05 \\ E-mail : geck@math.jussieu.fr
}

